\title{
Estimating Irregular Wave Runup on Rough, Impermeable Slopes
} US Army Corps of Engineers

by Steven A. Hughes

PURPOSE: The Coastal and Hydraulics Engineering Technical Note (CHETN) described herein provides a new formula for estimation of irregular wave runup on rough, impermeable slopes. The runup guidance is based on the wave momentum flux parameter described in CHETN-III-67 (Hughes 2003a). Sample calculations illustrate application of the formula.

BACKGROUND: Estimates of maximum wave runup on rough, impermeable sloping structures (riprap revetments) are necessary to determine whether overtopping will occur for a specified wave condition and water level. Design formulas were originally developed based on theory and smallscale laboratory experiments using regular waves. As laboratories acquired the capability to generate more realistic irregular waves, improved wave runup formulas were proposed based on wave parameters representative of the irregular wave train. However, unlike regular waves that result in a single value of maximum wave runup, irregular waves produce a runup distribution. Thus, it was necessary for the runup formulas to determine a representative parameter of the wave runup distribution. Presently, the most common irregular wave runup parameter is $R_{u 2 \%}$. This parameter is defined as the vertical distance between the still-water level (swl) and the elevation exceeded by 2 percent of the runup values in the distribution. In other words, for every 100 waves running up a slope, two waves would have a runup elevation exceeding the level estimated by $R_{u 2 \%}$.

Irregular wave runup design guidance for rough, impermeable slopes given in the Coastal Engineering Manual is based on runup measurements acquired during irregular wave rock armor stability experiments conducted at Delft Hydraulics and reported by van der Meer and Stam (1992). The guidance is presented as two empirical formulas with each formula valid over a specific range of Iribarren numbers. The formulas in the Coastal Engineering Manual for the 2-percent runup elevation are as follows:

$$
\frac{R_{u 2 \%}}{H_{m o}}=\left\{\begin{array}{lll}
0.96 \xi_{\text {om }} & \text { for } & 1.0<\xi_{\text {om }}<1.5 \\
1.17\left(\xi_{\text {om }}\right)^{0.46} & \text { for } & 1.5<\xi_{\text {om }}
\end{array}\right.
$$

with the Iribarren number defined as:

$$
\xi_{o m}=\frac{\tan \alpha}{\sqrt{\frac{H_{m o}}{L_{o m}}}}
$$


and

$$
\begin{aligned}
R_{u 2 \%} & =\text { vertical runup distance exceeded by } 2 \text { percent of wave runups } \\
H_{m o} & =\text { zeroth-moment energy-based significant wave height } \\
\xi_{o m} & =\text { deepwater Iribarren number based on mean period } T_{m} \\
L_{o m} & =\text { deepwater wavelength }\left[=(g / 2 \pi) T_{m}{ }^{2}\right] \\
g & =\text { gravitational acceleration } \\
T_{m} & =\text { mean wave period associated with wave spectrum } \\
\tan \alpha & =\text { structure slope }
\end{aligned}
$$

The mean wave period is used instead of the peak spectral wave period, $T_{p}$, in the runup formulas to accommodate different widths of the wave spectrum. However, in some cases, design wave conditions are specified in terms of $T_{p}$, so it is necessary to give an estimate of $T_{m}$. The ratio of $T_{p} / T_{m}$ varies for different spectrum types as follows:

$$
\frac{T_{p}}{T_{m}}= \begin{cases}1.15-1.27 & \text { for JONSWAP spectra } \\ 1.22-1.41 & \text { for Pierson - Moskowitz spectra }\end{cases}
$$

van der Meer and Stam (1992) gave the range $T_{p} / T_{m}=\xi_{o p} / \xi_{o m}=1.1-1.2$ when they converted Hunt's runup equation to use an Iribarren number based on peak spectral period, $\xi_{o p}$, instead of $\xi_{\text {om }}$. Using the median value in this range of $\xi_{o p} / \xi_{o m}=1.15$, Equation 2 can be converted to a form that uses $T_{p}$ rather than $T_{m}$, i.e.,

$$
\frac{R_{u 2 \%}}{H_{m o}}=\left\{\begin{array}{lll}
0.835 \xi_{o p} & \text { for } & 1.15<\xi_{o p}<1.72 \\
1.10\left(\xi_{o p}\right)^{0.46} & \text { for } & 1.72<\xi_{o p}
\end{array}\right.
$$

where

$$
\xi_{o p}=\frac{\tan \alpha}{\sqrt{\frac{H_{m o}}{L_{o p}}}}
$$

and $L_{o p}$ is the deepwater wavelength based on peak period, $T_{p}$.

Figure 1 presents the original data of van der Meer and Stam (1992) along with additional laboratory observations reported by Ahrens and Heimbaugh (1988). The observed values of dimensionless runup $\left(R_{u 2 \%} / H_{m o}\right)$ were plotted versus Iribarren number based on peak period $\left(\xi_{o p}\right)$ for structure slopes of 1:2, 1:3, and 1:4. The experiments of van der Meer and Stam were for structures with relatively deep water at the toe, and the maximum value of relative wave height was about $H_{m o} / h=0.25$. Ahrens and Heimbaugh's experiments used shallower water depths with the maximum value of relative wave height of about $H_{m o} / h=0.64$. The solid lines in Figure 1 were plotted using Equation 4. Generally, the empirical runup equations represent the data well. 


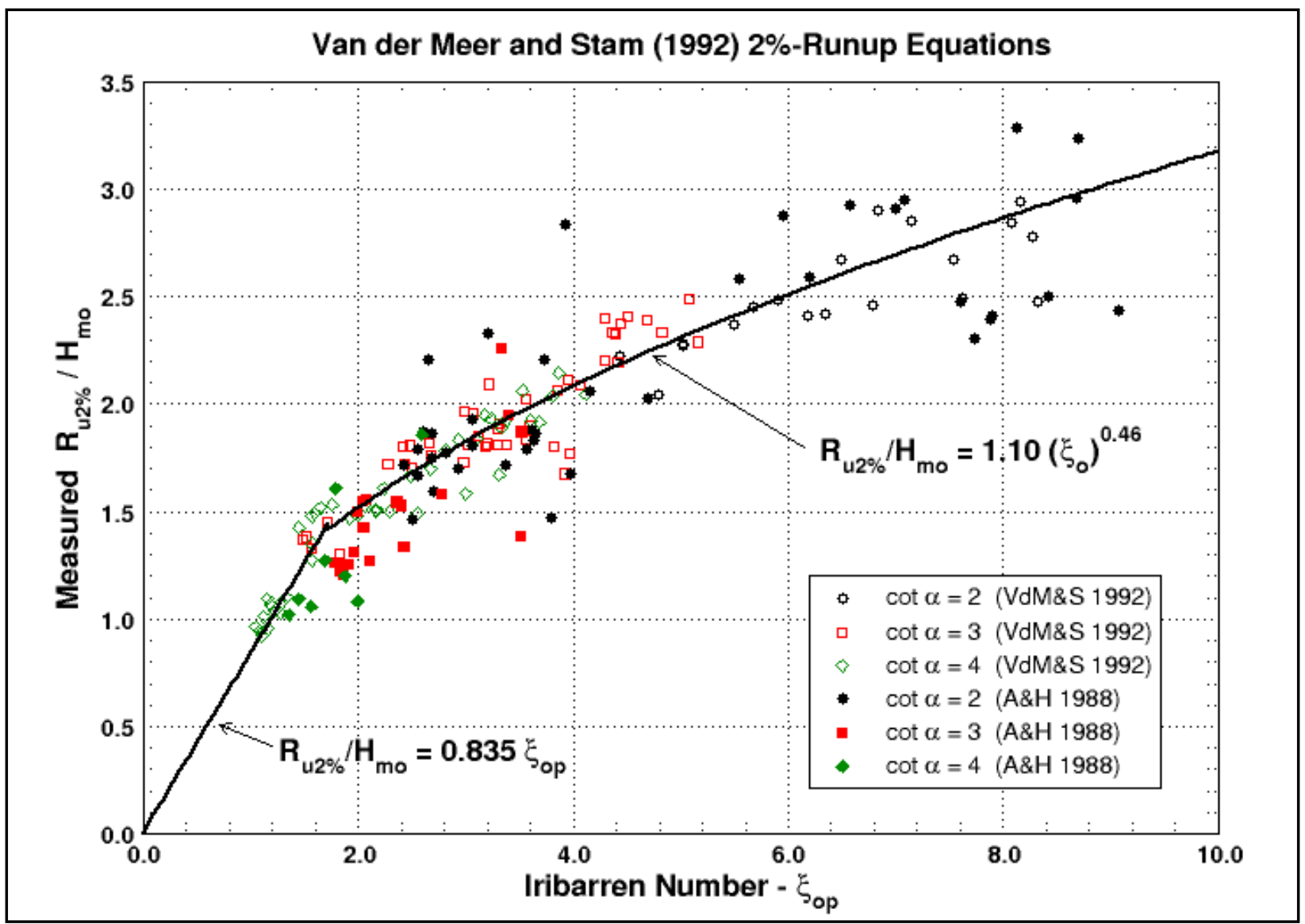

Figure 1. Irregular wave runup guidance given in the Coastal Engineering Manual for rough, impermeable slopes

New Wave Runup Equation: Hughes (2003a, 2004a) presented a new nondimensional parameter representing the maximum depth-integrated wave momentum flux that occurs in progressive water waves. The parameter, referred to as the wave momentum flux parameter $\left(P_{M F}\right)$, was defined as

$$
P_{M F}=\left(\frac{M_{F}}{\rho g h^{2}}\right)_{\text {max }}
$$

where

$$
\begin{aligned}
M_{F} & =\text { depth-integrated wave momentum flux } \\
\rho & =\text { fluid mass density } \\
g & =\text { gravitational acceleration } \\
h & =\text { water depth }
\end{aligned}
$$

Because $\left(M_{F}\right)_{\max }$ has units of force per unit wave crest length, it was argued that maximum depthintegrated wave momentum flux would provide a good characterization of wave processes at coastal structures. 
Hughes (2003a, 2004a) established an empirical equation for estimating the wave momentum flux parameter for finite amplitude, nonlinear waves based on a numerical solution technique (Fourier approximation). The resulting, purely empirical equation, was given as

$$
\left(\frac{M_{F}}{\rho g h^{2}}\right)_{\max }=A_{0}\left(\frac{h}{g T^{2}}\right)^{-A_{1}}
$$

where

$$
\begin{aligned}
& A_{0}=0.639\left(\frac{H}{h}\right)^{2.026} \\
& A_{1}=0.180\left(\frac{H}{h}\right)^{-0.391}
\end{aligned}
$$

and $H$ and $T$ are the regular wave height and period, respectively. More information and a sample calculation of wave momentum flux are given in CHETN-III-67 (Hughes 2003a).

Hughes (2003b, 2004b) derived a simple generic expression for wave runup in terms of wave momentum flux. He argued that the weight of fluid within the hatched wedge area (ABC) of Figure 2 is proportional to the maximum depth-integrated wave momentum flux of the wave just before it reached the toe of the structure slope. The resulting equation was in the form

$$
\frac{R}{h}=C \cdot F(\alpha)\left(\frac{M_{F}}{\rho g h^{2}}\right)^{1 / 2}
$$

where $R$ is the maximum vertical runup from swl, and $C \cdot F(\alpha)$ is an unknown function of structure slope angle. For convenience the subscript "max" has been dropped from the wave momentum flux parameter.

In the Equation 9 runup equation, relative runup $(R / h)$ is directly proportional to the square root of the wave momentum flux parameter. (Note that representing the runup sea surface slope as a straight line is an approximation and may not be fully appropriate for nonbreaking waves on steep slopes where the water surface has pronounced curvature.)

Applying Equation 9 to irregular wave runup requires that regular wave height and period $(H$ and $T)$ used to estimate the wave momentum flux parameter using Equations 6, 7, and 8 be replaced with representative irregular wave parameters $\left(H_{m o}\right.$ and $\left.T_{p}\right)$. This substitution does not imply that an equivalence exists between values of wave momentum flux parameter calculated for regular and irregular waves; it only provides a convenient standard for application with irregular waves when establishing empirical relationships. For irregular waves, always check that the depth-limited wave height does not exceed the rule-of-thumb $H_{m o} \leq 0.6 \mathrm{~h}$. 


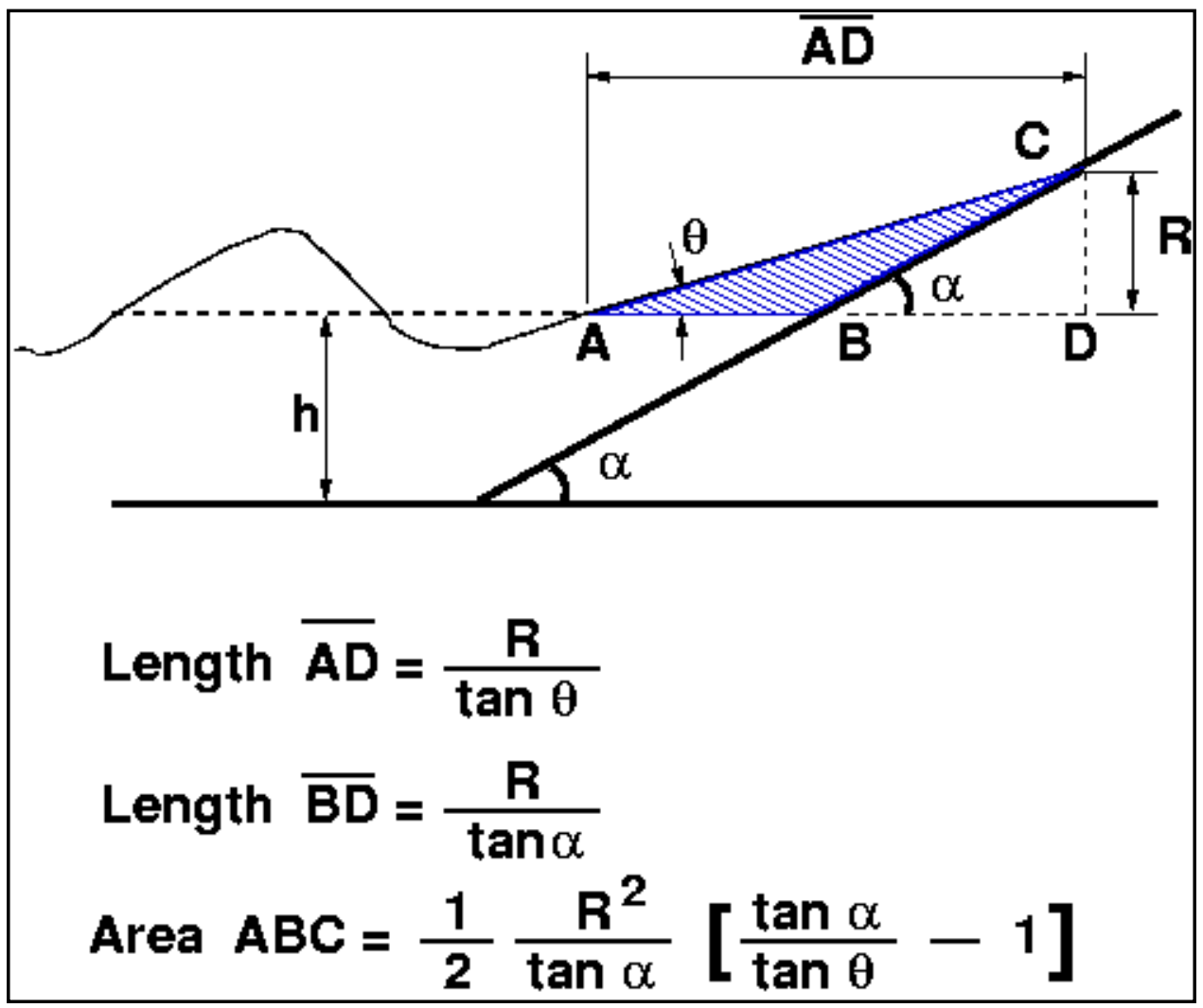

Figure 2. Maximum wave runup on smooth impermeable plane slope

Summary of Irregular Wave Runup Prediction for Smooth Slopes: Hughes (2003b) recommended the following equations for prediction of irregular wave runup on smooth, impermeable slopes for nonbreaking (surging/collapsing) waves and breaking (plunging/spilling) waves as delineated by values of $H_{m o} / L_{p}$.

Nonbreaking (surging/collapsing) waves $\left(H_{m o} / L_{p}<0.0225\right)$ :

$$
\frac{R_{u 2 \%}}{h}=1.75\left[1-e^{-(1.3 \cot \alpha)}\right]\left(\frac{M_{F}}{\rho g h^{2}}\right)^{1 / 2} \quad \text { for } \quad 1.0 \leq \cot \alpha \leq 4.0
$$

Breaking (plunging/spilling) waves $(\mathrm{Hmo} / \mathrm{Lp}>0.0225)$ :

$$
\frac{R_{u 2 \%}}{h}=1.75\left[1+e^{-(0.47 \cot \alpha)}\right]\left(\frac{M_{F}}{\rho g h^{2}}\right)^{1 / 2} \quad \text { for } \quad 1.5 \leq \cot \alpha \leq 4.0
$$

The formulas are for structure slopes steeper than 1:4 $(\cot \alpha=4)$.

More recently Hughes (2004b) modified Equation 11 for breaking (plunging/spilling) waves to include irregular runup on smooth, impermeable slopes as mild as 1:30. The newer equation was given as: 


\section{Newer equation for breaking (plunging/spilling) waves $\left(H_{m o} / L_{p}>0.0225\right)$ :}

$$
\frac{R_{u 2 \%}}{h}=4.4(\tan \alpha)^{0.7}\left(\frac{M_{F}}{\rho g h^{2}}\right)^{1 / 2} \quad \text { for } \quad 1.5 \leq \cot \alpha \leq 30
$$

Equation 12 covers a much broader range of slopes, but it is slightly less accurate than Equation 11 when applied to structure slopes in the range $1.5 \leq \cot \alpha \leq 4.0$. Nevertheless, having one equation that predicts irregular wave runup over such a wide range of slopes is convenient, and it supports the simple concept used to derive the runup equation.

Irregular Wave Runup Prediction for Rough, Impermeable Slopes: Slope roughness will reduce the 2-percent runup level predicted using the equations for smooth, impermeable slopes (Equations 10 and 11). One engineering approach is to multiply the runup estimates for smooth, impermeable slopes by a reduction factor to account for various types of slope roughness. The Coastal Engineering Manual contains reduction factors as summarized by de Waal and van der Meer (1992) based on Dutch experience, and the reduction factor for rock and riprap structures ( 1 and 2 layers) varies between 0.5 and 0.6 . Rock and riprap structures impede runup not only by slope roughness, but also by permeability of the riprap and any underlayers placed over the impermeable slope. Voids are constantly filling and draining with the wave runup/rundown cycle, and the effect of riprap permeability will vary with wave period. Thus, using a single constant to represent runup reduction associated with rough, impermeable riprap slopes is simplistic. However, this approach is justified by the success of past runup estimation formulas.

The original riprap slope runup data of van der Meer and Stam (1992) and Ahrens and Heimbaugh (1988) were plotted as a function of the wave momentum flux parameter to ascertain any difference between breaking (plunging and spilling) waves on the slope and nonbreaking (surging and collapsing) waves. No strong trend was evident for rough slopes, whereas a distinct difference was apparent for runup on smooth slopes (Hughes 2003b, 2004b). Because the majority of the laboratory data represented waves that break on the slopes, the newer runup equation (Equation 12) for plunging/spilling waves was used to estimate an appropriate reduction factor for runup on rough, impermeable slopes. This method is appealing because it retains the conceptual runup model shown in Figure 2. The best-fit reduction factor representing the mean of the data was 0.505 , which is at the lower end of the reduction factor range for rock-armored slopes given by de Waal and van der Meer (1992).

Applying the reduction factor to Equation 12 results in the following new equation for estimating irregular wave runup on rough (riprap) impermeable slopes for both breaking and nonbreaking incident wave conditions.

New equation for irregular wave runup on rough, impermeable slopes:

$$
\frac{R_{u 2 \%}}{h}=4.4(\tan \alpha)^{0.7}\left(\frac{M_{F}}{\rho g h^{2}}\right)^{1 / 2}(0.505) \quad \text { for } \quad 2.0 \leq \cot \alpha \leq 4.0
$$

Figure 3 compares estimated values of relative runup $\left(R_{u 2} \% / h\right)$ to measured values. Reasonable correspondence was expected because these were the same data used to determine the appropriate 
reduction factor. The data of van der Meer and Stam (1992) are clustered at low values of $R_{u 2 \%} / h$ because the experiments were conducted for structures in relatively deep water. In deeper water, incident wave nonlinearity is less, and the waves bear more resemblance to sinusoids.

A direct comparison between the new runup formula (Equation 13) and formulas given in the Coastal Engineering Manual (Equation 4) for the two laboratory data sets is made in Figures 4 and 5 . The new runup equation based on wave momentum flux (Figure 4) gave a reasonable estimate of the measured 2-percent runup, but the van der Meer and Stam (1992) formulas, converted to use peak period, $T_{p}$, gave slightly better results (Figure 5). Most of the difference between the two methods stems from data obtained for the 1:2 rough slope, particularly the data of van der Meer and Stam (1992) that appear to be entirely nonbreaking waves with all values of $\xi_{\text {op }}>4.4$.

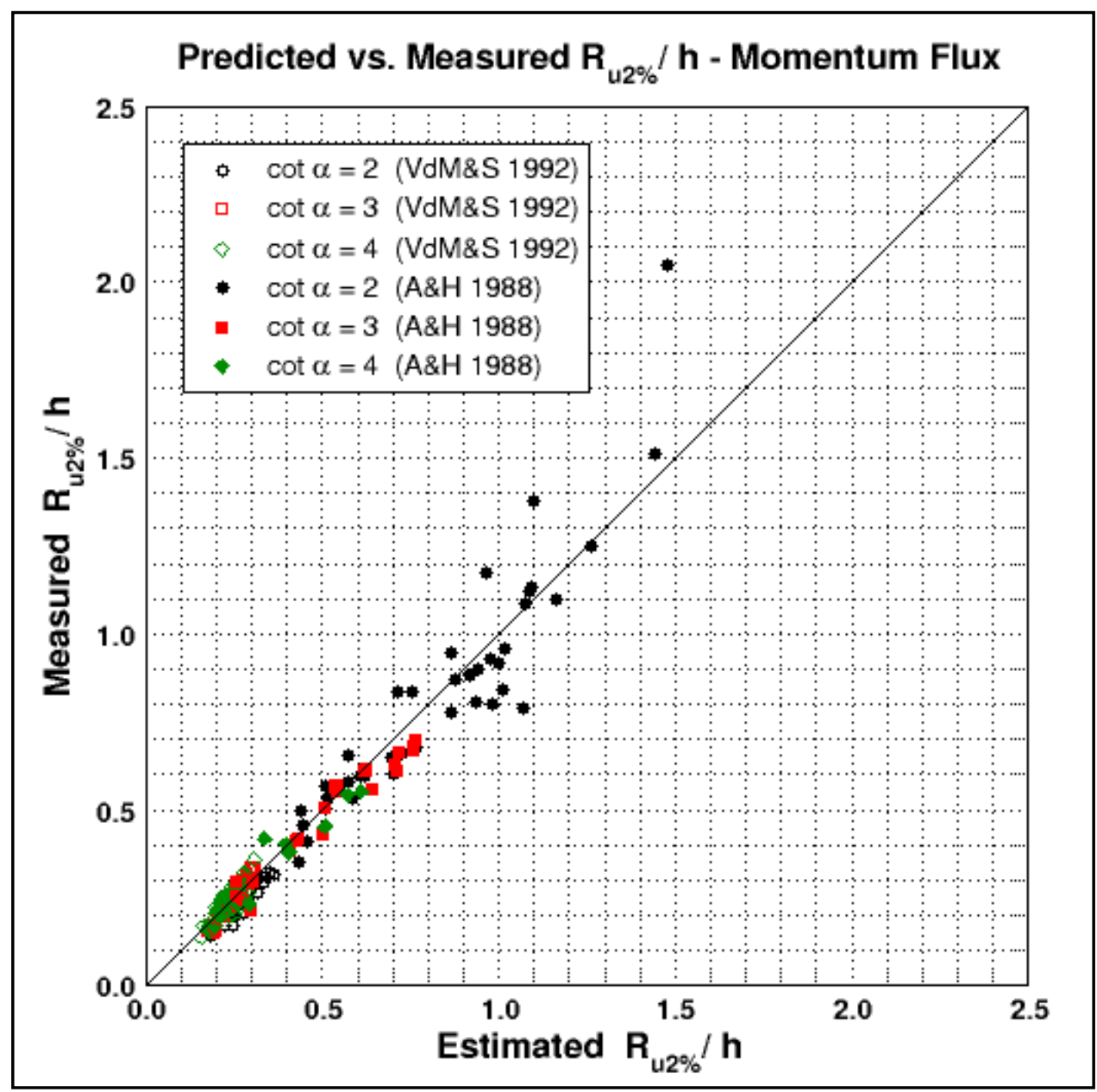

Figure 3. Predicted versus measured $R_{u 2 \%} / h$ for rough, impermeable plane slopes 


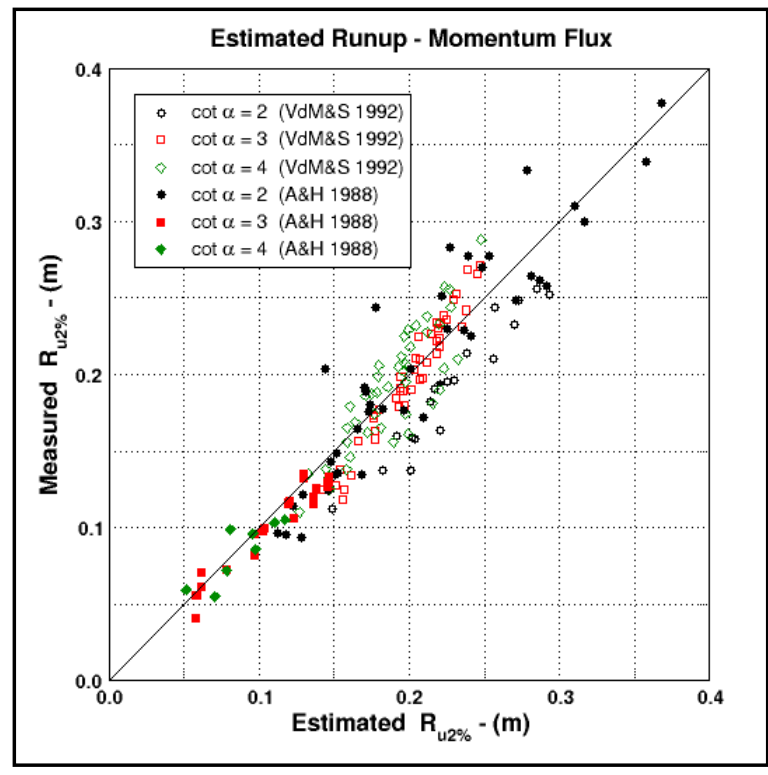

Figure 4. Runup estimation using wave formulas momentum flux formula (Eq. 13)

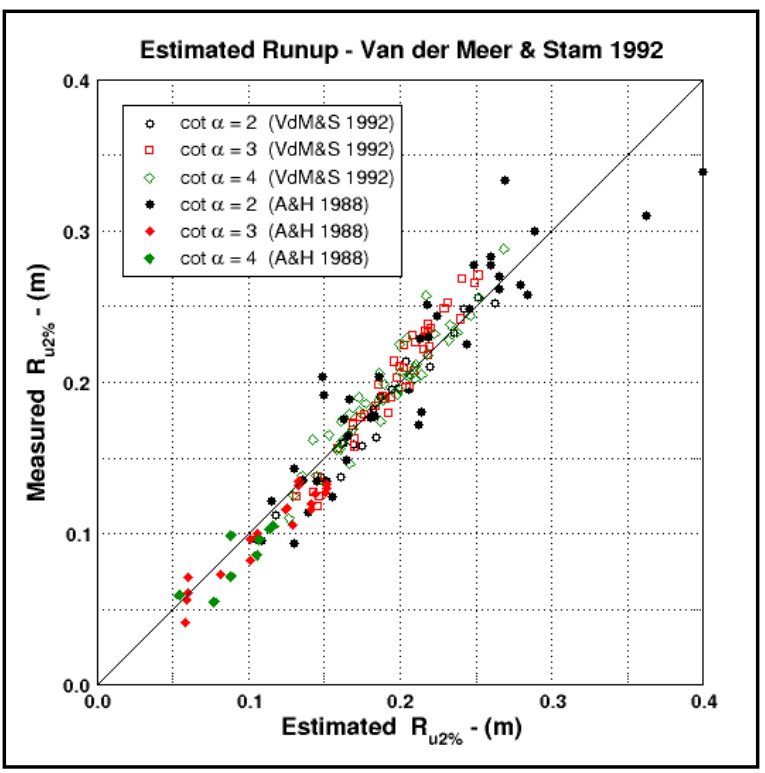

Figure 5. Runup estimation using Coastal Engineering Manual converted to use peak period, $T_{p}$ (Eq. 4)

van der Meer and Stam (1992) determined their empirical runup equation by a direct curve fit to the data. This procedure was able to represent the nonbreaking wave data better than the method of applying a uniform reduction factor to the estimates for the corresponding runup on smooth slopes using the plunging/spilling runup equation based on wave momentum flux. The nonbreaking wave runup data were isolated and fitted with a separate curve, and this improved the wave momentum flux estimates to equal those of van der Meer and Stam (1992). However, the improvement was not significant enough to justify having two formulas when a single formula gives estimates comparable to those of van der Meer and Stam.

\section{EXAMPLE: IRREGULAR WAVE RUNUP ON ROUGH, IMPERMEABLE SLOPES:}

Find: The vertical runup distance from the swl which is exceeded by only 2 percent of the waves (i.e., $\left.R_{u 2 \%}\right)$ for structure slopes of $1: 2$ and $1: 4(\tan \alpha=0.5$ and 0.25$)$.

\section{Given:}

$$
\begin{array}{rlrl}
h & =20 \mathrm{ft}^{1} \quad & - \text { water depth at toe of slope } \\
T_{p} & =9.0 \mathrm{sec} \quad-\text { wave period associated with spectral peak } \\
H_{m o} & =8 \mathrm{ft} \quad-\text { zeroth-moment significant wave height } \\
g & =32.2 \mathrm{ft} / \mathrm{sec}^{2}-\text { gravitational acceleration } \\
\tan \alpha & =0.5,0.25 \quad-\text { structure slope }
\end{array}
$$

\footnotetext{
1 To convert feet to meters, multiply number of feet by 0.3048 .
} 
Calculate wave momentum flux parameter: First calculate values of relative wave height and relative depth as

$$
\frac{H}{h}=\frac{8 \mathrm{ft}}{20 \mathrm{ft}}=0.4 \quad \text { and } \quad \frac{h}{g T^{2}}=\frac{20 \mathrm{ft}}{\left(32.2 \mathrm{ft} / \mathrm{sec}^{2}\right)(9 \mathrm{sec})^{2}}=0.0077
$$

Next, find the values of the coefficient $A_{0}$ and $A_{1}$ from Equations 7 and 8, respectively, i.e.,

$$
\begin{aligned}
& A_{0}=0.639\left(\frac{H}{h}\right)^{2.026}=0.639(0.4)^{2.026}=0.0998 \\
& A_{1}=0.180\left(\frac{H}{h}\right)^{-0.391}=0.180(0.4)^{-0.391}=0.2576
\end{aligned}
$$

Finally, the nondimensional wave momentum flux parameter is calculated from Equation 6 as

$$
\left(\frac{M_{F}}{\rho g h^{2}}\right)_{\max }=A_{0}\left(\frac{h}{g T^{2}}\right)^{-A_{1}}=0.0998(0.0077)^{-0.2576}=0.35
$$

\section{Calculate runup for 1:2 slope:}

For rubble-armored impermeable slopes, the appropriate runup equation for both surging/collapsing and plunging/spilling breaker types is given by Equation 13 as

$$
\frac{R_{u 2 \%}}{h}=4.4(\tan \alpha)^{0.7}\left(\frac{M_{F}}{\rho g h^{2}}\right)^{1 / 2}(0.505)
$$

First check that the structure slope falls within the range of applicability for Equation 13, i.e.,

$$
\cot \alpha=\frac{1}{\tan \alpha}=\frac{1}{0.5}=2.0
$$

which is within the range of $2.0 \leq \cot \alpha \leq 4.0$.

The nondimensional relative 2-percent runup is found as:

$$
\frac{R_{u 2 \%}}{h}=4.4(\tan \alpha)^{0.7}\left(\frac{M_{F}}{\rho g h^{2}}\right)^{1 / 2}(0.505)=4.4(0.5)^{0.7}(0.35)^{1 / 2}(0.505)=0.81
$$

and the dimensional 2-percent runup is

$$
R_{u 2 \%}=0.81 h=0.81(20 \mathrm{ft})=16.2 \mathrm{ft}
$$


For comparison, the present Coastal Engineering Manual method given by Equation 4 estimates runup to be $R_{u 2 \%}=15.9 \mathrm{ft}$.

\section{Calculate runup for $1: 4$ slope:}

After checking that the 1:4 structure slope falls within the range of applicability for the plunging/ spilling wave runup equation, the nondimensional relative 2-percent runup is found from Equation 13 as

$$
\frac{R_{u 2 \%}}{h}=4.4(\tan \alpha)^{0.7}\left(\frac{M_{F}}{\rho g h^{2}}\right)^{1 / 2}(0.505)=4.4(0.25)^{0.7}(0.35)^{1 / 2}(0.505)=0.50
$$

and the dimensional 2-percent runup is

$$
R_{u 2 \%}=0.50 h=0.50(20 \mathrm{ft})=10.0 \mathrm{ft}
$$

The present Coastal Engineering Manual method given by Equation 4 estimates runup to be $R_{u 2 \%}=11.5 \mathrm{ft}$.

SUMMARY: This CHETN has described a new empirical formula for estimating the vertical runup distance above the swl that will be exceeded by only 2 percent of the irregular wave runups on rough, impermeable slopes. The formula is based on the same hypothesis used in an earlier analysis of runup on smooth, impermeable slopes (Hughes 2003b, 2004b), i.e., the weight of water above swl at maximum runup is proportional to the maximum depth-integrated wave momentum flux occurring in a wave just before it reaches the toe of the impermeable plane slope.

The irregular wave runup data on rough/riprap slopes of Ahrens and Heimbaugh (1988) and van der Meer and Stam (1992) were analyzed in terms of the nondimensional wave momentum flux parameter. There was only slight difference in runup between waves that broke on the slope (plunging and spilling breakers) and nonbreaking (surging and collapsing) waves. The laboratory data were well represented by applying a constant reduction factor to the corresponding equation for runup on smooth, impermeable slopes. The new formula (Equation 13) has reasonable predictive capability for irregular wave runup on slopes in the range 1:2 to 1:4. An example calculation illustrates application of the runup equation.

POINTS OF CONTACT: This CHETN is a product of the Coastal Inlet Structures work unit of the Coastal Inlets Research Program (CIRP) being conducted at the U.S. Army Engineer Research and Development Center, Coastal and Hydraulics Laboratory. Questions about this technical note can be addressed to Dr. Steven A. Hughes (Voice: 601-634-2026, Fax: 601-634-3433, email: Steven.A.Hughes@erdc.usace.army.mil).For information about the Coastal Inlets Research Program (CIRP), please contact the CIRP Technical Leader, Dr. Nicholas C. Kraus at Nicholas.C.Kraus@erdc.usace.army.mil. Beneficial reviews were provided by Dr. Jeff Melby, Coastal and Hydraulics Laboratory; and Mr. John Ahrens, retired Coastal and Hydraulics Laboratory 
and Sea Grant. Special thanks to Mr. John Ahrens and Dr. Jentsje van der Meer, INFRAM, for providing their original irregular wave runup data.

\section{REFERENCES}

Ahrens, J. P., and Heimbaugh, M. S. 1988. “Approximate upper limit of irregular wave runup on riprap,” Technical Report CERC-88-5, U.S. Army Engineer Waterways Experiment Station, Coastal Engineering Research Center, Vicksburg, MS.

de Waal, J. P., and van der Meer, J. W. (1992). "Wave runup and overtopping on coastal structures," Proceedings of the 23rd International Coastal Engineering Conference, American Society of Civil Engineers, 2, 1758-1771.

Headquarters, U.S. Army Corps of Engineers. (2001). Coastal Engineering Manual, EM 1110-2-1100, Washington, DC.

Hughes, S. A. (2003a). "Wave momentum flux parameter for coastal structure design," ERDC/CHL CHETN-III-67, U.S. Army Engineer Research and Development Center, Vicksburg, MS.

Hughes, S. A. (2003b). "Estimating irregular wave runup on smooth, impermeable slopes," ERDC/CHL CHETN-III-68, U.S. Army Engineer Research and Development Center, Vicksburg, MS.

Hughes, S. A. (2004a). "Wave momentum flux parameter: A descriptor for nearshore waves," Coastal Engineering. Elsevier, 51(11), 1067-1084.

Hughes, S. A. (2004b). "Estimation of wave run-up on smooth, impermeable slopes using the wave momentum flux parameter," Coastal Engineering. Elsevier, 51(11), 1085-1104.

van der Meer, J. W., and Stam, C. M. (1992). "Wave runup on smooth and rough slopes of coastal structures," Journal of Waterway, Port, Coastal, and Ocean Engineering, American Society of Civil Engineers, 118(5), 534-550.

NOTE: The contents of this technical note are not to be used for advertising, publication, or promotional purposes. Citation of trade names does not constitute an official endorsement or approval of the use of such products. 